\title{
Latest Result from XMASS
}

\author{
K. Kobayashi ${ }^{1,2}$ \\ ${ }^{1}$ Kamioka observatory, Institute of Cosmic Ray Research, the University of Tokyo, Higashi-Mozumi, Kamioka, Hida, Gifu, 506-1205, Japan \\ ${ }^{2}$ Kavli Institute for the Physics and Mathematics of the Universe (WPI), the University of Tokyo, Kashiwa, Chiba, 277-8582, Japan
}

\begin{abstract}
XMASS is a multi-purpose experiment using a single-phase liquid-xenon scintillator detector located underground at Kamioka Observatory in Japan. The primary aim is to detect dark matter signal. We have taken data from November 2013 to February 2019, for more than five years. With this long-term data, we have conducted not only dark matter searches, but also various researches in particle and astroparticle physics. We report standard WIMPs, annual modulation, hidden photons and axio-like particles, and exotic neutrinos searches. No significant signals are observed in these searches.
\end{abstract}

Keywords: dark matter, xenon, neutrino, millicharge, underground

DOI: 10.31526/ACP.NDM-2020.5

\section{INTRODUCTION}

The XMASS project aims at detecting dark matter, $p p$ and ${ }^{7}$ Be solar neutrinos, and neutrinoless double beta decay using large volume of pure liquid xenon. The first physics goal of the XMASS experiment is to detect dark matter. Various astronomical observations provide strong evidence for a large amount of dark matter in the universe. However, its nature is still unknown. Weakly interacting massive particles (WIMPs) are dark matter candidates motivated by theories beyond the Standard Model. Although many direct dark matter search experiments have looked for WIMPs, but dark matter have not been observed yet. This project searches for nuclear recoils in liquid xenon caused by WIMPs. Since the XMASS detector is sensitive not only to nuclear recoils but also electron recoils, various candidates of dark matter other than WIMPs are also considered. In addition, we searched for exotic neutrino interactions using solar neutrinos.

\section{XMASS DETECTOR}

The XMASS detector is located at the Kamioka mine, 1000m underneath the top of Mt. Ikenoyama (i.e. $2700 \mathrm{~m}$ water equivalent underground) in Japan. The detector consists of two components, the inner and outer detectors (ID and OD, respectively). The ID is equipped with 642 inward-facing photomultiplier tubes (PMTs) in an approximate spherical shape in a copper vessel filled with pure liquid xenon. The entire structure is immersed in liquid xenon. The amount of liquid xenon in the sensitive region is $832 \mathrm{~kg}$. A PMT photo coverage of more than $62 \%$ is achieved and the PMT quantum efficiency at the scintillation wavelength of liquid xenon $(175 \mathrm{~nm})$ is more than $28 \%$. The vessel which holds liquid xenon and the PMT holder is made of oxygen free high conductivity copper (OFHC) and the size is $1.12 \mathrm{~m}$ in diameter. To reduce the amount of liquid xenon, an OFHC copper filler is installed in the gap between the PMT holder and the inner vessel. The vessel is covered with another vessel for vacuum insulation. The ID is installed at the centre of the OD, which is a cylindrical water tank (10 $\mathrm{m}$ in height and $10 \mathrm{~m}$ in diameter) with seventy two 20 -inch PMTs. The OD is used as an active shield for cosmic ray muons and a passive shield for low-energy gamma rays and neutrons. Data taking has been perfomed from November 2013 to February 2019.

\section{PHYSICS RESULTS FROM XMASS}

We have searched for various kinds of dark matters with different methods. We report three of them, that are standard WIMP search, annual modulation search, and hidden photon/axion-like particle search. Also we report exotic neutrino interaction search.

\subsection{Standard WIMP search}

We have searched for standard WIMPs, which is our primary aim to detect, using 705.9 live days of data in a fiducial volume containing $97 \mathrm{~kg}$ of liquid xenon at the center of the detector. We uses the events after passing through our standard cut, fiducial volume cut using photo electron based and timing based vertex reconstruction. The events with reconstructed energy greater than 2 $\mathrm{keV}_{e e}$ are used. The event rate in the fiducial volume after the data reduction was $(4.2 \pm 0.2) \times 10^{-3} \mathrm{day}^{-1} \mathrm{~kg}^{-1} \mathrm{keV}_{e e}^{-1}$ at $5 \mathrm{keV}_{e e}$, with a signal efficiency of $20 \%$. All the remaining events are consistent with our background evaluation based on independent assays of background radioactivities and a simultaneous fitting of signal and background, shown in Figure 1. Most of the backgrounds come from the mis-reconstructed events originated from ${ }^{210} \mathrm{~Pb}$ in the copper plates lining the detector's inner surface. In the study of ${ }^{210} \mathrm{~Pb}$ in copper using an alpha counter [2], we found that even OFHC contain a few tens $\mathrm{mBq} / \mathrm{kg}$ of ${ }^{210} \mathrm{~Pb}$ in bulk. In the OFHC 
in XMASS, ${ }^{210} \mathrm{~Pb}$ concentration is estimated to be $25 \pm 5 \mathrm{mBq} / \mathrm{kg}$, that is consistent to the measurement. Figure 2 shows the spinindependent WIMP nucleon cross section limit. The obtained limit was $2.2 \times 10^{-44} \mathrm{~cm}^{2}$ for a WIMP mass of $60 \mathrm{GeV} / c^{2}$ at the $90 \%$ confidence level (CL), which was the most stringent limit among results from single-phase liquid xenon detectors [3].

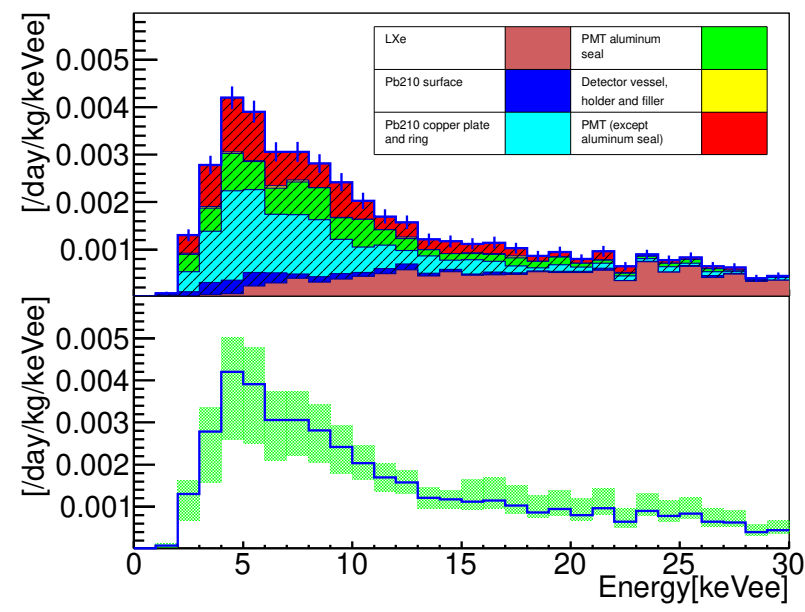

FIGURE 1: Expected background from MC study. Upper figure shows the breakdown. Lower figure shows total expected background (blue line) with systematic errors (green region).

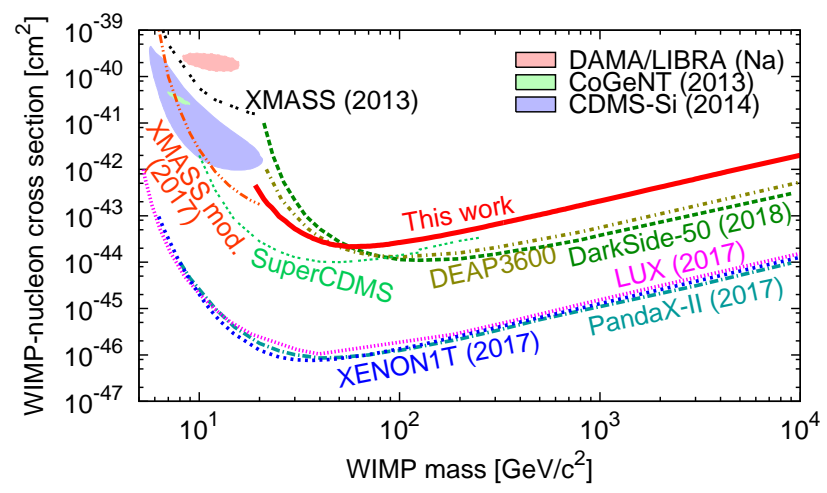

FIGURE 2: The spin-independent WIMP-nucleon cross section limit as a function of theWIMP mass at the $90 \%$ CL. The XMASS limit is shown by the solid line compared with other experimental results.

\subsection{Annual modulation search}

An annual modulation signal coming from the earth orbiting around the sun would be one of the indications of dark matter signal. We use 800 live days of data with $832 \mathrm{~kg}$ target mass to search. Simple event selection is performed instead of vertex reconstruction in order to use lower energy events than that of standar WIMP search. However, no sigificant signal excess was found. Assuming WIMP dark matters elastically scattering on the xenon target, the exclusion upper limit for the WIMP-nucleon cross section was $1.9 \times 10^{-41} \mathrm{~cm}^{2}$ at $8 \mathrm{GeV} / c^{2}$ at $90 \%$ CL. For the model-independent case, without assuming any specific dark matter model, we obtained consistent results with the null hypothesis with a p-value of 0.11 in the $1-20 \mathrm{keV}$ energy region. This search probed this region with an exposure that was larger than that of DAMA/LIBRA. We also did not find any significant amplitude in the data for modulations with period between 50 and 600 days in the energy region between 1 to $6 \mathrm{keV}$ [4].

\subsection{Hidden photons and axion-like particles search}

Hidden photons and axion-like particles are candidates for cold dark matter if they were produced non-thermally in the early universe. We conducted a search for both of these bosons using 800 live days of data with $327 \mathrm{~kg}$ of liquid xenon in the fiducial volume. Expected peak in number of photo electron distribution is searched for in 590-1760 PE region corresponding to 40-120 keV. No significant signal was observed, and thus we set constraints on the strength of the kinetic mixing of hidden photons [5], $\alpha^{\prime} / \alpha$, 
and the coupling constant $\mathrm{g}_{A e}$ of axion-like particles in the mass range from 40 to $120 \mathrm{keV} / \mathrm{c}^{2}$, resulting in $\alpha^{\prime} / \alpha<6 \times 10^{26}$ and $\mathrm{g}_{A e}<4 \times 10^{13}$. These limits are the most stringent derived from both direct and indirect searches to date. The detail is shown in Ref. [5].

\subsection{Exotic neutrino interaction search}

The XMASS detector is also able to explore other physics topics effectively due to their low backgrounds (BGs) and low energy threshold. Since the flux of solar neutrino is large, we searched for exotic neutrino interactions, such as neutrino millicharge, neutrino magnetic moment, and neutrino interactions due to dark photons using solar neutrinos. In the neutrino millicharge search, we applied the same criteria as standard WIMP search. However, we found no significant signal excess and accordingly we set an upper limit for neutrino millicharge of $5.1 \times 10^{-12} e$ at the $90 \%$ CL. Figure 3 shows the data and the best-fit signal + BG MC with the signal MC at the $90 \%$ CL upper limit. This limit is for neutrinos, not antineutrinos, and for neutrinos it is more stringent than the previous limit by more than three orders of magnitude. Though the originally emitted solar neutrinos are $v_{e}$, the neutrinos arriving at the Earth consist of all the three flavors, with the other flavors produced by neutrino oscillations: At Earth $54 \pm 2 \%$ are $v_{e}, 23 \pm 1 \%$ are $v_{\mu}$, and $23 \pm 1 \%$ are $v_{\tau}$. Using this, we set upper limits for each flavor to be $7.0 \times 10^{-12} e$ for $v_{e}, 1.1 \times 10^{-11} e$ for $v_{\mu}$, and $1.1 \times 10^{-11} e$ for $v_{\tau}$. Figure 4 compares our result with those of other experiments. The limits for $v_{\mu}$ and $v_{\tau}$ are the best limits also including antineutrinos.

Using the 2-200 keV energy range, we also searched for a signal excess due to neutrino magnetic moment and neutrino interaction due to dark photons, but again no significant excesses are found. The 90\% CL upper limit for the neutrino magnetic moment is estimated from the $\chi^{2}$ probability density function to be $\mu_{v}=1.0 \times 10^{-10} \mu_{B}$. The upper limits for $g_{B-L}$ for $M_{A^{\prime}}=1 \times 10^{-3} \mathrm{MeV} / c^{2}$, and $10 \mathrm{MeV} / c^{2}$ are $1.4 \times 10^{-6}$ and $9.3 \times 10^{-5}$ at $90 \% \mathrm{CL}$, respectively.

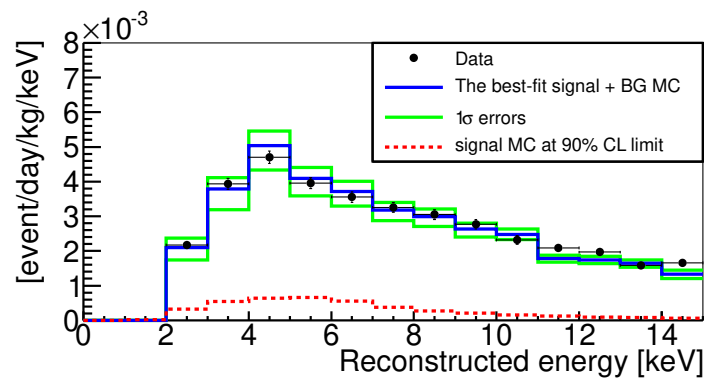

FIGURE 3: The energy distribution after applying all the cuts. The black points show the data. The blue histogram shows the best-fit signal + BG MC simulation with $1 \sigma$ errors shown by the green histograms. The red-dotted histogram shows the $90 \%$ CL upper limit for the neutrino-millicharge signal.

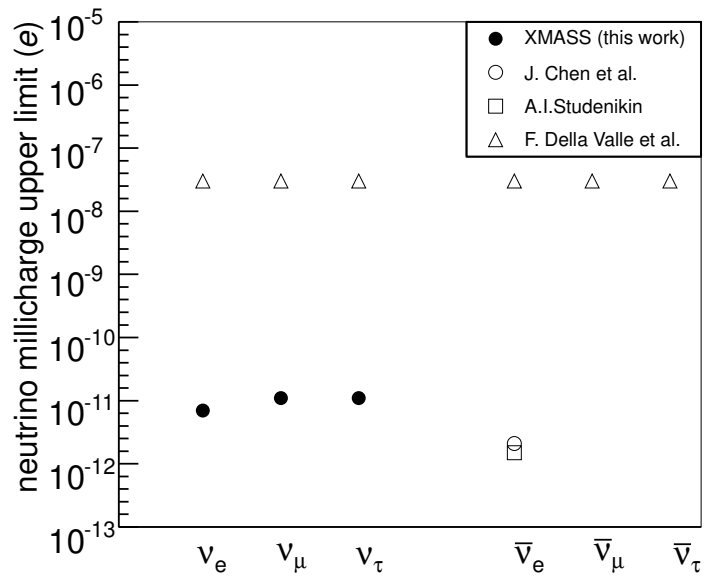

FIGURE 4: 90\% CL upper limits of neutrino millicharge for each flavor in ours and other experiments [6, 7,8]. The limit from F. Della Valle et al. [8] is for neutrino masses of less than $10 \mathrm{meV}$.

\section{SUMMARY}

XMASS have taken data from November 2013 to February 2019. Three kinds of dark matter searches using the XMASS data are shown. No significant signals of dark matters are found. Moreover, we searched for exotic neutrino interactions such as neutrino 
millicharge, neutrino magnetic moment, and neutrino interactions due to dark photons. No siginificant signal exdesses are found. In the neutrino millicharge search, we set a neutrino millicharge upper limit of $5.1 \times 10^{-12} e$ at $90 \%$ CL. It is three orders of magnitude better than the best previous limit [8].

We are analyzing data for other dark matter searches and other physics topics as well. We keep searching for dark matter in XMASS.

\section{ACKNOWLEDGEMENTS}

We also gratefully acknowledge the cooperation of Kamioka Mining and Smelting Company. This work was supported by the Japanese Ministry of Education, Culture, Sports, Science and Technology, Grant-in-Aid for Scientific Research, ICRR Joint-Usage, JSPS KAKENHI Grant Number, 26104004, and 17K05465, 19 H05805.

\section{References}

[1] K. Abe et al. (XMASS collaboration), Nucl. Instr. and Meth. A 716, (2013) 78-85.

[2] K. Abe et al. (XMASS collaboration), Nucl. Instr. and Meth. A 884, (2018) 157-161.

[3] K. Abe et al. (XMASS collaboration), Phys. Lett. B 789, (2019) 45-53.

[4] K. Abe et al. (XMASS collaboration), Phys. Rev. D97, 102006 (2018).

[5] K. Abe et al. (XMASS collaboration), Phys. Lett. B 787, (2018) 153-158.

[6] A. Studenikin, Europhys. Lett. 107 (2014) 39901.

[7] J. Chen et al., Phys. Rev. D 90 (2014) 011301.

[8] F. Della Valle et al., Euro. Phys. J. C76 (2016) 24. 